\title{
Posterior Indirect Adhesive Restorations: for Vital Posterior Teeth: Clinical Report
}

\author{
Imen Kalghoum, Hajjemi Hayet, Belhssan Harzallah, Mounir cherif and Dalenda Hadyaoui* \\ Department of Fixed Prosthodontics, Research Laboratory of Occlusodontics and Ceramic Prostheses LR16ES15, Faculty of Dental Medicine, \\ University of Monastir, Monastir, Tunisia
}

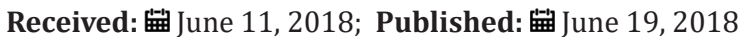

*Corresponding author: Dalenda Hadyaoui, Department of Fixed Prosthodontics, Research Laboratory of Occlusodontics and Ceramic Prostheses LR16ES15, Faculty of Dental Medicine, University of Monastir, Monastir, Tunisia

\begin{abstract}
This article describes a case of compromised posterior vital teeth treated by indirect bonded ceramic restorations. A 24-yearold female patient presented to the department of Prosthodontics. She was complaining about sensitivity in the posterior region. A comprehensive examination revealed defective amalgam restoration in the first molar with compromised cusps. The treatment plan included indirect bonded ceramic restorations consisting of on-lay on the concerned teeth.
\end{abstract}

Keywords: Ceramic inlay-onlay; bonding, esthetic; vital posterior teeth; minimally invasive approach; minimally invasive approach

\section{Introduction}

The daily use of indirect bonded restoration is very frequent in case of extended coronal destruction of vital posterior tooth [1]. The major treatment goal of compromised vital posterior teeth is minimal invasiveness based on adhesion which have made operator's strategies completely changing in favor of minimally invasive bonded restorations. The principal advantage of those restoration is that preparation allows a greater preservation of healthy tissue [2]. Several studies evaluating the clinical performance of ceramic inlays and onlay showed a high survival rate around 96.7\%. According to Reiss [3], during a period of 12 year only $8 \%$ cases of failure were observed. In the same context $S$ Jorgen reported, $89 \%$ of survival rate at 10 years with increased failure rate on non-vital teeth. On these bases, ceramic onlay and inlays are today scientifically recognized restorations for the posterior region [4]. Qual trough and Wilson stressed, also, the role of the bonding procedure to the overall success of these ceramic restorations [5]. Some authors reported that, the success of those restorations is the result of a careful evaluation of the indications, a design of the preparation suitable for the situation and the right choice of ceramic material [6,7]. When an indirect restoration with inlay-onlay is determined to be the best treatment option for vital posterior tooth, the clinician must then determine the geometric configuration of the cavity preparation and if there will be a cusps coverage which remains a controversial point [8]. Cavity design should follow principles of adaptation, resistance and retention [9]. This case report describes the innovative technique that outlines the reconstruction of damaged posterior teeth with lithium disilicate ceramic inlay-onlay.

\section{Clinical report}

A 30 year old male patient presented to the department of Prosthodontics. He was suffering from a dental sensitivity in the posterior region. After a comprehensive examination, failing amalgam restoration in the first and second right molars were evident and recurrent decay was noted. The second molar presented a MOD amalgam restoration whereas the first molar presented compromised cusps (Figure1). The margins of the cavities were supra gingivally placed. The failed amalgam, the recurrent decay was removed, and temporary restorations were placed on during the first Appointment (Figure 2) and bonded ceramic restorations were planned. The first step was to mark the patient's occlusion with an articulating paper. Tooth preparation was performed with medium-grit diamonds. The depth of the cavity was $1.5 \mathrm{~mm}$ with rounded occluso axial angles. Absence of undercuts with a cervical axial wall convergence of $10^{\circ}$ to $20^{\circ}$ (According to the manufacturer's axis of insertion (Figure 3) Whereas, Dentin close to the pulp was covered with ionomer glass cement (Build up or block out) with internal rounded corner (Figure 4) and the margins were finished. The temporization was performed using a silicon 
index and acrylic resin (Texton) and cemented with a temporary a non-eugenol cement. Impression of both upper and lower arch was made using light body and heavy body putty material (Silicon) (Figure 5). The restorations were manufactured from etcheable ceramic bulks (Lithuim discilicate, IPS e max cad) (Figures 6 \& 7). For a durable adhesive interface, optimal dry field conditions are required. A clean surface and careful execution of the bonding procedure according to the manufacturer's instructions are also prerequisites for success the use of rubber dam was necessary. The thickness of the cuspal coverage in onlay was measured using a pair of tactile compasses. The internal surfaces of the all-ceramic inlayOnlay were etched with 5\% hydrofluoric acid (IPS Ceramic etching gel, Ivoclar Vivadent) for 2 minutes, the external surface should be waxed in order to protect it from etching, then silanated with Monobond-S (Ivoclar Vivadent) and allowed to air dry.
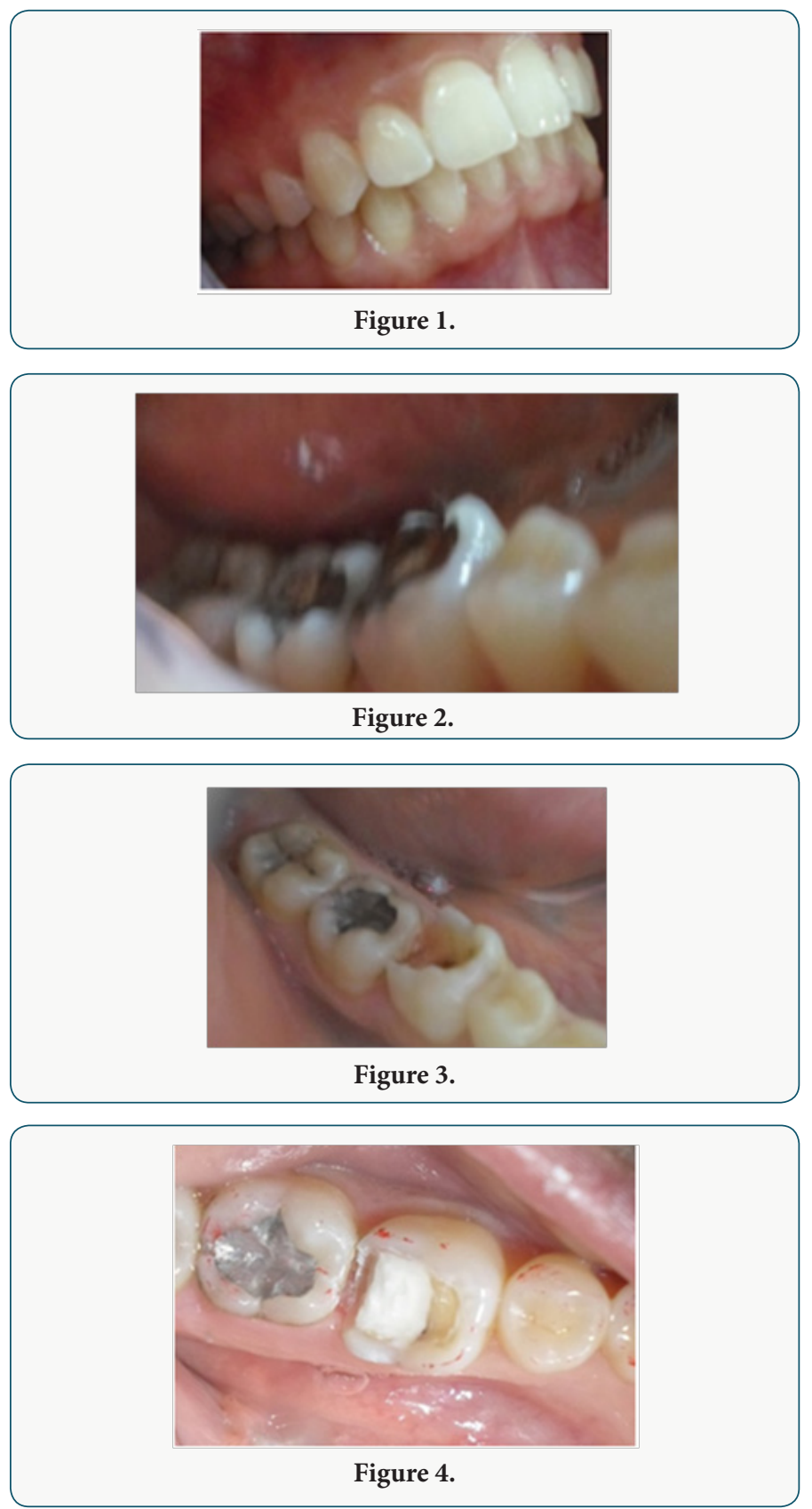
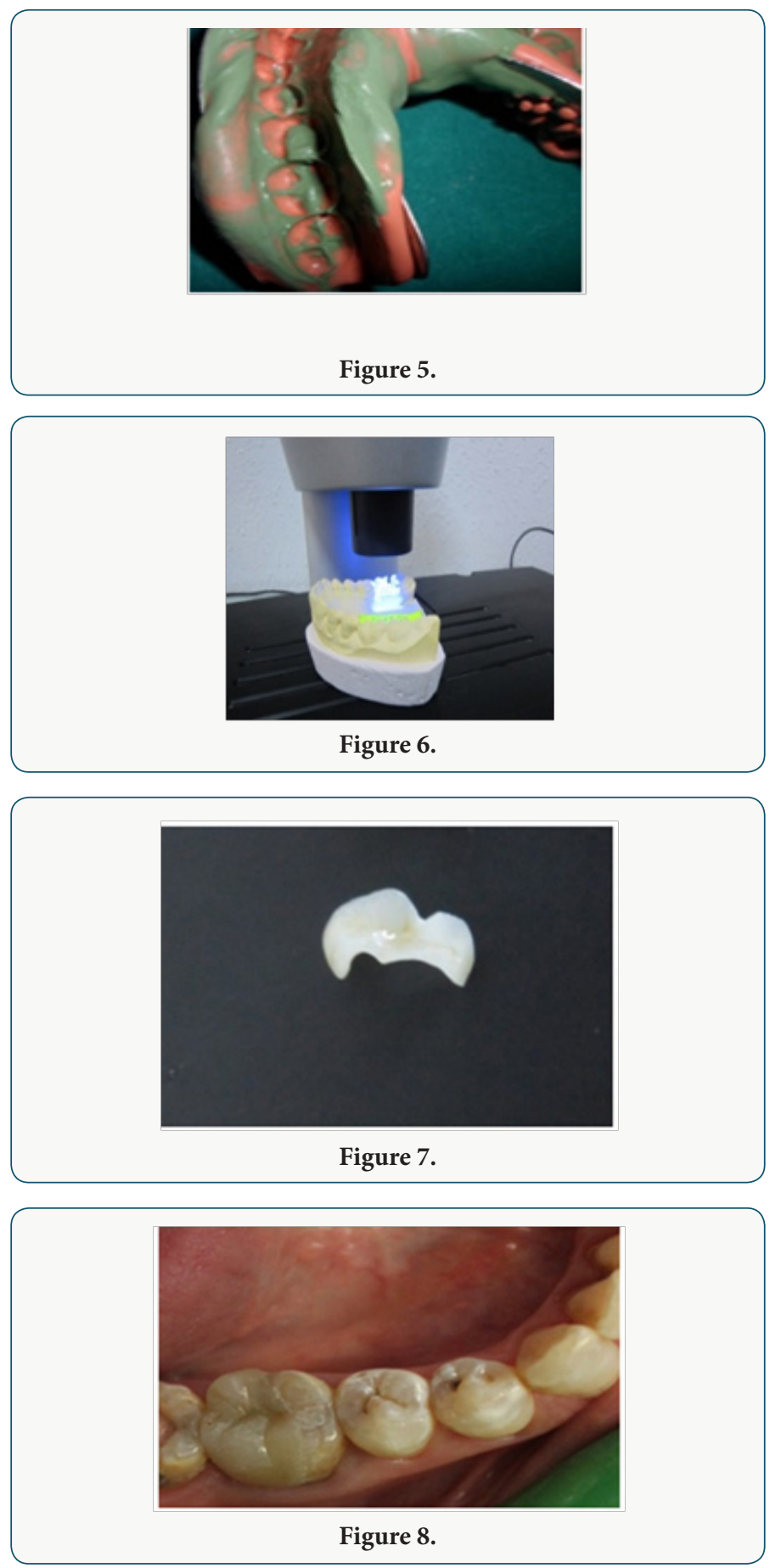

The preparation surfaces were cleaned with special paste. The total-etching technique was used for the condition of tooth surfaces with $37 \%$ phosphoric acid gel for 30 seconds, then rinsed off. Resin luting agent is then applied to the preparation and the all ceramic restoration. The inlay-onlay was seated and excess luting material was removed. Gross excess resin can be removed after a spot cur. The restoration should be supported while the resin is cured. Light curing is then done in accordance with the resin manufacturer's recommendations. The occlusion and the articulation were checked carefully after the inlay-onlay was luted in order to avoid fracture (Figure 8). Patient was recalled after 6 months for follow up in 
order to evaluate color, marginal adaptation, abrasion, secondary caries, fracture and post-operative pain.

\section{Discussion}

Indirect bonded all ceramic restorations are considered as an attractive alternative for Class I and II amalgam. They become highly recommended for treating vital posterior teeth especially when excessive width cavities and fractures may preclude the use of direct posterior composite restorations. They are stronger than composite resins with superior physical properties. Their clinical effectiveness is strongly related to the development of dental ceramics as well as bonding systems. $[10,11]$. In our case the use of lithium disilicate was extremely recommended. In fact, this material has been tested after the simulation of 5 years of wear. In comparison to other ceramics and even enamel in the study, the wear of lithium disilicate was very low, showing a sound surface without any wear of opposing dentition [12]. From an esthetic standpoint, the lithium disilicate material is very versatile. The opacity is controlled by the nanostructure of the material [13]. The restoration in our case, it was indistinguishable from the tooth being restored, after bonding. The morphologies of preparation can be different, depending on clinical situation, but there are some general rules to apply: Hence, the preparation should have smooth flowing margins to facilitate the fabrication of the restoration [14]. Box walls should converge in an occlusion direction. This will facilitate optical capture, Nevertheless, this rule was not completely respected. In order to achieve a balance between the preservation of tooth structure and strength of the material and in order to avoid fracture caused by insufficient material thickness, authors concluded that the idealized inlay preparation design requires a cavity depth of between 1.5 and $2 \mathrm{~mm}$; cavity width of $1 / 3$ the intercuspal distance; and a total occlusal convergence angle about $20^{\circ}$ and rounding of all internal line angles. Finally, to ensure a secure bonding with a durable result the margins should be supragingivally located and when an indirect restoration is demanded the cavities should not be extended below the enamel-cement joint $[15,16]$.

\section{Conclusion}

Among all the treatment options available for aesthetic intracoronal restorations, ceramic inlay-onlay have performed reasonably well in clinical situations, when a vital posterior tooth is compromised because of wide preparations, compared with other materials. Nevertheless, they are extremely technique sensitive and the indication should be considered carefully, and further experience is necessary before they can be generally recommended for clinical use. There does not appear to be any one ceramic onlay technique or material which clearly provides clinical performance superior to the others, however the use of IPS max lithium disilicate offers new opportunities in restorative dentistry. The material's strength and optical properties offer multiple options for achieving highly durable and esthetically pleasing restorations.

\section{References}

1. Rocca GT, Krejci I (2007) Bonded indirect restorations for posterior teeth : From cavity preparation to provisionalization. Quitessence int 38(5): 371-379.

2. Roulet JF, Soderholm KJM, Longmate (1995) Effects of treatment and storage conditions on ceramic/composite bond strength. J Dent Res 74(1): 381-387.

3. Shen C, Oh WS, Williams JR (2004) Effect of post-silanization drying on the bond strenght of composite to ceramic. J Prosthet Dent 91(5): 453458.

4. Monticelli F, Toledano M, Osorio R, Ferrari M (2006) Effect of temperature on the silane coupling agents when bonding core resin to quartz fiber posts. Dent Mater 22(11): 1024-1028.

5. Silva RHBT, Ribeiro APD, Catirze ABCE (2009) Clinical performance of indirect - onlay for posterior teeth after 40 months. Braz J Oral Sci 8(3): 154

6. Barghi N, Berry T, Chung K (2000) Effects of timing and heat treatment of silanated porcelain on the bond strength. J Oral Rehabil 27(5): 407412 .

7. Fligor J (2008) Preparation design and considerations for direct posterior composite inlay-onlay restoration. Practical procedures and aesthetic dentistry 20(7): 413-419.

8. Felden A, Shmalz G, Federlin M (1998) Retrospective clinical investigation and survival analysis on ceramic inlays and partial ceramic crowns: results up to 7 years. Clin Oral Invest 2(4): 161-167.

9. Saridag SS, Sevimay M, Pekkan G (2013) Fracture resistance of teeth restored with all-ceramic inlays and onlays after eight years. Oper Dent 38(6): 626-634.

10. Kramer N, Frankenberger R (2005) Clinical performance of banded leucite-reinforced glass ceramic inlays and onlays after eight years. Dent Mater 21(3): 262-271.

11. Kramer N, Ebert J, Petschelt (2006) A Ceramic inlays bonded with two adhesives after 4 years. Dental materials 22(1): 13-21.

12. Hopp CD, Land MF (2013) Considerations for ceramic inlays in posterior teeth; a review. Clinical cosmetic and investigation Dentistry 18(5): 2132.

13. Fligor J (2008) Preapration design and considerations for direct posterior composite inlay-onlay restoration. Pratical procedures and aesthetic dentistry 20(7): 413-419.

14. Abraham S, Attur K, Singh SKN (2011) Aesthetic Inlays. International journal of dental clinics 3(3): 62-64.

15. Thompson MC, Thompson KM, Swain (2010) The all-ceramic, inlay supported fixed partial denture.Part 1.Ceramic inlay preparation design: a literature review. Aust Dent J 55(2): 120-127.

16. Ahlers MO, Land MF (2013) Considerations for ceramic inlays in posterior teeth: a review. Clinical cosmetic and investigational Dentistry 5: 21-32. 
This work is licensed under Creative Commons Attribution 4.0 License

To Submit Your Article Click Here: Submit Article

DOI: 10.32474/IPDOAJ.2018.01.000123

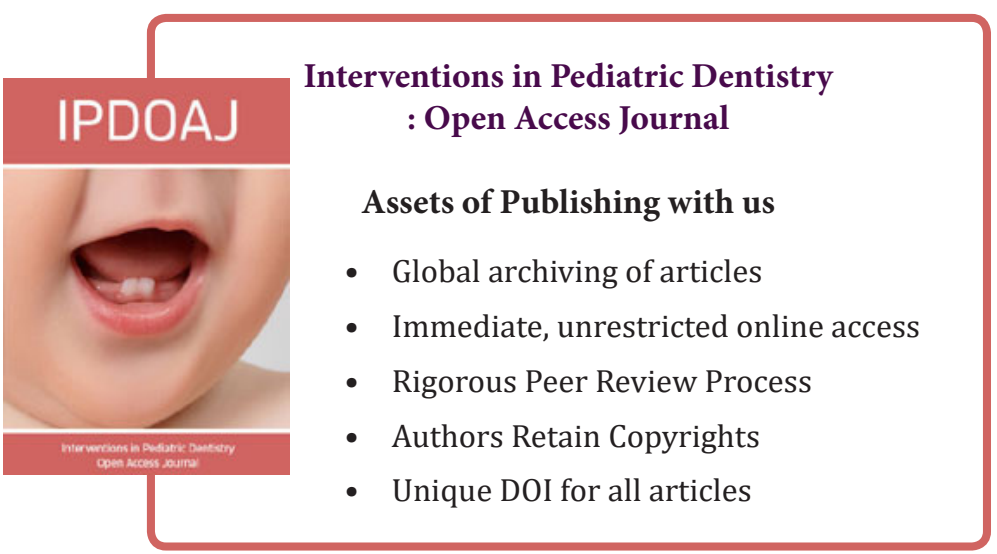

